\title{
Advances in biotechnology for fibre processing
}

\author{
Georg M. Guebitz • Johanna Buchert • \\ Artur Cavaco-Paulo · Elisabeth Heine
}

Published online: 3 June 2006

(C) Springer Science+Business Media B.V. 2006

From 2000 to 2005 the European Union COST Action 847 on Textile Quality and Biotechnology has investigated the potential of biotechnology in the processing of fibres. Members of the network from 32 countries have coordinated more than 20 European projects focusing on biotechnical and enzymatic technologies in the processing of cellulose- and protein-based materials and have recently focused on the functionalization of synthetic polymers. Based on the scientific outcome of these projects, experts have concluded, at the 4th COST 847 Congress in Gran Canaria, Spain, from February 20-23, 2005, that biotechnology plays an important role in the development of environmentally friendly production technologies in textile processing and in strategies to improve the final product quality. Advances in genetic

Preface by Guest Editors of Special Issue

G. M. Guebitz $(\bowtie)$

Graz University of Technology, Graz, Austria

e-mail: guebitz@tugraz.at

J. Buchert

VTT Biotechnology, Espoo, Finland

A. Cavaco-Paulo

University of Minho, Braga, Portugal

E. Heine

German Wool Research Institute at Aachen

University of Technology (DWI), Aachen, Germany engineering, combined with a better understanding of enzyme structure and function on polymeric materials, have opened up new possibilities for functionalization of fibre materials and/or for improving environmental aspects in processing. Based on the oral contributions of this congress, this Special Issue of Biotechnology Letters provides an overview on recent advances in this area.

New hydrolases have been isolated which can partially hydrolyze the surface of polyester-based fibres thereby improving their hydrophilicity and further finishing steps (Alisch et al. 2006-loc. cit.). Transglutaminases have been used for crosslinking of gelatine-based materials used in tissue engineering (Bertoni et al. 2006). Besides such enzymatic methods for the functionalization of polymers, genetic engineering of protein-based polymers can be used to design complex and highly functional macromolecules with applications in the biomedical area (Arias et al. 2006). Despite the high potential of proteases for antishrinking and anti-felting treatment, as well as washing of wool based textiles, partial hydrolytic degradation of the fibres has prevented the use of proteases. Several approaches including chemical modification of enzymes are presented here which can target protease action to the surface of the fibres (Lenting et al. 2006; Zhang et al. 2006; Erlacher et al. 2006; Vasconcelos et al. 2006).

Chemical modification of enzymes can also be used to diminish decolorization of fabric-bound 
dyes by laccases directing their action to solubilized dyes and thereby preventing dye transfer in washing processes (Schroeder et al. 2006). Besides their potential to decolorize dyes, the ability of laccases to cross-couple phenolic molecules has been exploited in enzyme-assisted dyeing of cotton (Hadzhiyska et al. 2006). For the treatment of dyeing effluents, a combination of an anaerobic/aerobic treatment has shown promising results (Lourenco et al. 2006). Finally, recent advances in the development of enzymebased processes for the biopreparation of cotton and bast fibres are shown (Csiszar et al. 2006; Kozlowski et al. 2006).

Future developments of biotechnology in fibre processing seem to be going in two directions: on the one hand, exploitation of new enzymes and genetic engineering towards highly functional polymers and fibre based materials with highvalue applications, e.g. in the biomedical area, will continue. On the other hand, the mechanistic understanding on enzyme/polymer interactions, combined with advances in chemical and genetic enzyme engineering, will also lead to implementation of environmentally friendly technologies in processing of low-cost fibre materials.

Finally, we would like to thank all scientists and representatives from industry who have contributed to the COST 847 Network during the past 5 years and thereby, promoted progress in the area of textile biotechnology. We also thank Sonja Heumann for her brilliant assistance during the organization of this special issue. 